\title{
Muon physics: from phenomenology to experiment
}

\author{
Ana M. Teixeira* \\ Laboratoire de Physique de Clermont (UMR 6533), CNRS/IN2P3, \\ Univ. Clermont Auvergne, 4 Av. Blaise Pascal, F-63178 Aubière Cedex, France \\ E-mail: ana.teixeira@clermont.in2p3.fr
}

\begin{abstract}
New Physics can be manifest at the high intensity frontier through a vast array of phenomena, which includes rare transitions and decays (forbidden in the Standard Model), or tiny deviations from the Standard Model's expectation. Muons are very powerful and versatile probes of New Physics - numerous experiments rely on the study of "muon channels" to search for processes violating lepton number, charged lepton flavours, or even the universality of lepton flavours, as well as CP violation in the lepton sector. Following a short overview of the status of dedicated experimental searches, we comment on how muon observables can shed light on the underlying model of New Physics at work.
\end{abstract}

The 21st international workshop on neutrinos from accelerators (NuFact2019) August 26 - August 31, 2019

Daegu, Korea

${ }^{*}$ Speaker. 


\section{Introduction: leptonic observables and signals of New Physics}

Several observational problems, as well as theoretical issues, suggest the need to consider New Physics (NP) beyond the Standard Model (SM). Interestingly, several lingering tensions between the SM's predictions and observation (typically at the level of 2-3 $\sigma$ ) appear to be nested in leptonic observables: this is the case of the anomalous magnetic moment of the muon (and more recently that of the electron), as well as the lepton flavour universality violating (LFUV) hints arising from $B$-meson decays.

The SM, in its original formulation, conserves both individual lepton flavours and total lepton number; electric dipole moments (EDMs) arise from the CKM phase (at the 4-loop level) and are extremely tiny. In the most minimal SM extension allowing to accommodate neutrino oscillation phenomena (via the addition of right-handed Dirac neutrinos), neutral lepton flavours are violated in charged current interactions, as encoded in the $U_{\mathrm{PMNS}}$ mixing matrix. In such a minimal framework, total lepton number is still conserved and interactions remain lepton flavour universal. Transitions violating charged lepton flavours are possible, but the associated rates are suppressed by the smallness of light neutrino masses, thus lying beyond experimental sensitivity; although contributions to the EDMs now emerge at 2-loop level, these also lie beyond experimental reach. The observation of any of these processes thus clearly signals the presence of a genuinely NP contribution. Muons are uniquely versatile probes of NP. Due to the advent of very intense beams [1], they can be produced in copious amounts, and be used to search for deviations from the SM's expectations, or for very rare processes - in particular phenomena which are either forbidden or extremely suppressed in the SM. After a very brief overview of several muon properties and muonic observables sensitive to NP, we illustrate how these can help constraining and reconstructing the underlying model of NP.

\section{Muon observables and experimental status}

In this section we briefly summarise the current status and future experimental prospects in what concerns several classes of (muon) observables: EDMs, anomalous magnetic lepton moments, lepton flavour universality violation (LFUV) and charged lepton flavour violation (cLFV).

\subsection{Leptonic moments: EDMs and $(g-2)_{\ell}$}

Electric dipole moments are sensitive probes of new sources of $\mathrm{CP}$ violation. For electrons, increasingly stronger bounds have been obtained from paramagnetic atoms (as $\mathrm{Tl}, \mathrm{Cs}$ ) and molecules $\left(\mathrm{HfF}^{+}, \mathrm{ThO}, \ldots\right)$. Following the recently obtained bound, $\left|d_{e}\right| / e \lesssim 1.1 \times 10^{-29} \mathrm{~cm}$ [2], the ACME collaboration expects to improve the sensitivity by a factor of 10-20 [3]. The muon EDM is a purely leptonic observable, offering a direct probe of NP. The current bound $\left|d_{\mu}\right| / e \lesssim 1.8 \times 10^{-19} \mathrm{~cm} \mathrm{[4]}$ is expected to be improved by two orders of magnitude at J-PARC and FNAL (in association with the corresponding $(g-2)_{\mu}$ programmes); at PSI, future prospects for a compact storage ring should allow a sensitivity $d_{\mu}<5 \times 10^{-23}$ e.cm (single $\mu$ mode) [3].

The anomalous magnetic moment of the muon is an example of a longstanding tension between observation and SM's prediction (whose most important sources of uncertainty remain associated with the hadronic vacuum polarisation and hadronic light-by-light contributions). The 
comparison between the BNL experimental measurement [5] and SM prediction lead to deviations between $3.7 \sigma[6]$ and $3.3 \sigma$ [7]. In the near future, the E989 experiment at FNAL is expected to clarify the situation (see [8]). Recent improvements in the SM computation of the anomalous magnetic moment of the electron, together with a new determination of the fine structure constant $\alpha$ (from Cs atoms), have led to the emergence of a new tension in association with $(g-2)_{e}$, currently at the level of $2.5 \sigma$ [9]. Curiously, the two tensions on $(g-2)_{e, \mu}$ also hint that the NP at their origin is further responsible for the violation of LFU: lepton universality suggests that the tensions should scale as $\Delta a_{e} / \Delta a_{\mu} \approx m_{e}^{2} / m_{\mu}^{2} \sim+2.4 \times 10^{-5}$, while from observation one has $\Delta a_{e}^{\exp } / \Delta a_{\mu}^{\exp } \sim-3.3 \times 10^{-4}$.

\subsection{LFUV in meson decays}

Several observables signaling LFUV (between electrons and muons) have been studied, in particular concerning (semi)leptonic meson decays. In the kaon sector, LFUV can be tested by the comparison of the helicity suppressed widths through the ratio $R_{K}^{\ell}=\frac{\Gamma(K \rightarrow e \bar{v})}{\Gamma(K \rightarrow \mu \bar{v})}$, with $R_{K}^{\ell \mathrm{SM}}=$ $(2.477 \pm 0.001) \times 10^{-5}[10]$ and $R_{K}^{\text {eexp }}=(2.488 \pm 0.009) \times 10^{-5}$ [11]. In the near future, TREK at J-PARC and NA62 are expected to reduce current errors to 2.5 per mille and sub-per mille level, respectively [3], thus strongly constraining the small window for NP contributions. Similar studies have been carried for pion decays. In the $B$-meson sector, hints for LFUV have emerged in association with the ratios $R_{K^{(*)}}=\frac{\mathrm{BR}\left(B \rightarrow K^{(*)} \mu^{+} \mu^{-}\right)}{\mathrm{BR}\left(B \rightarrow K^{(*)} e^{+} e^{-}\right)}$and $R_{D^{(*)}}=\frac{\mathrm{BR}\left(B \rightarrow D^{(*)} \tau^{-} \bar{v}\right)}{\mathrm{BR}\left(B \rightarrow D^{(*)} \ell^{-} \bar{v}\right)}$. Current measurements suggest tensions around $\mathscr{O}(2.5 \sigma-3 \sigma)$, a situation which will be hopefully clarified by coming experimental runs (Belle II and LHCb).

\section{3 cLFV transitions: the muon channels}

Several "muonic channels" offer the possibility to look for cLFV: the processes include radiative decays, three body decays, and several transitions occuring in the presence of muonic bound states. The current bound on radiative muon decays has been obtained by the MEG Collaboration at PSI, $\mathrm{BR}(\mu \rightarrow e \gamma)<4.2 \times 10^{-13}$ [12]. In the future, MEG II is expected to improve the sensitivity to $6 \times 10^{-14}$ [13]. The best limit on cLFV three-body muon decays $\operatorname{BR}(\mu \rightarrow 3 e)<1.0 \times 10^{-12}$ [14] is expected to be significantly improved in the coming years by the Mu3e collaboration at PSI to around $10^{-15}$ [15] (or even down to $10^{-16}$, should very high-intensity muon beams become available). "Muonic atoms" are formed when negatively charged muons are stopped in a target, and cascade down in energy until forming 1 s bound states. A cLFV neutrinoless muon-electron conversion, $\mu^{-}+(A, Z) \rightarrow e^{-}+(A, Z)$, can then occur. For spin-independent decays (coherent process) $)^{1}$, the rate generaly grows with increasing atomic number, being maximal for $30 \leq Z \leq 60$ [17]. The best limit has been obtained for Gold targets, $\mathrm{CR}(\mu-e, \mathrm{Au})<7 \times 10^{-13}$ [18]; in the future, several experiments will be dedicated to looking for muon-electron conversion [3]: DeeMe aims at reaching a sensitivity of $10^{-14}$ for $\mathrm{SiC}$ targets; working with Aluminium targets, Mu2e at Fermilab expects to reach $3 \times 10^{-17}$, while at JPARC the goal of the COMET experiment is to reach $10^{-15(-17)}$ in its Phase I (II). The muonic atom can also decay into a pair of electrons, $\mu^{-} e^{-} \rightarrow e^{-} e^{-}[19,20]$, a process whose decay rate is strongly enhanced by the Coulomb interaction between the muon and the electron wave functions, scaling with the atomic number as $(Z-1)^{3}$ (or even more, especially in the case of very large nuclei). This observable has not yet been experimentally searched for, but

\footnotetext{
${ }^{1}$ For a recent discussion of spin-dependent contributions to $\mu-e$ conversion, see [16].
} 
could be included in the physics programmes of COMET and of Mu2e. Muonium (bound states of positively charged muons and electrons, $\mu^{+} e^{-}$) [21] can also undergo cLFV oscillations and decays. At present, upper bounds have been established - but only for the conversion probability $\mathrm{P}(\mathrm{Mu}-\overline{\mathrm{Mu}})<8.3 \times 10^{-11}[22]$.

\subsection{Lepton number violation}

The violation of total lepton number (LNV) - in addition to a clear signal of NP - further reveals the presence of new Majorana states responsible for mediating the transition (or decay). In addition to neutrinoless double beta decays $(0 v 2 \beta), \mathrm{LNV}$ processes are being actively looked for at the high intensity frontier: these include (semi)leptonic meson and tau decays, as well as very rare muonic atom decays. The latter process corresponds to $\mu^{-}+(A, Z) \rightarrow e^{+}+(A, Z-2)^{*}$, in which the final state nucleus can also be in an excited state (denoted by an asterisk). The current bound (obtained by the SINDRUM Collaboration) is $\mathrm{CR}\left(\mu^{-}+\mathrm{Ti} \rightarrow \mathrm{e}^{+}+\mathrm{Ca}^{(*)}\right) \lesssim 3.6 \times 10^{-11}\left(1.7 \times 10^{-12}\right)$ [23]; in the future, both COMET and Mu2e could also search for the LNV muonic atom decay.

\section{The probing power of muons: (re)shaping New Physics}

Numerous extensions of the SM can give rise to contributions to the observables mentioned in the previous section; the results (be them in the form of new bounds or a signal) will certainly play a role in constraining several NP models, possibly helping to understand the nature of the new interactions at work.

\subsection{Muon observables: hints on the New Physics source of cLFV}

In general, beyond the SM (BSM) scenarios can be at the source of significant cLFV contributions via the introduction of new sources of flavour violation (corrections to SM vertices, or new flavour violating interactions) and/or new currents. While for radiative decays the dipole operator at its origin is necessarily realised at loop level, $\mu \rightarrow 3 e$ decays and neutrinoless conversion in matter can stem from both higher order (photon-, Z- or Higgs-mediated diagrams, and boxes) or even from tree level processes! In order to illustrate the discussion, let us focus on (generic) NP contributions to $\mu-e$ conversion (see also [24]). While the actual size of these contributions depends upon the properties of a given New Physics model, certain generic features can be infered by considering the following new terms in the BSM Lagrangian

$$
\mathscr{L}_{\phi}=g_{\ell \ell^{\prime}}^{\phi} \bar{\ell}^{\prime} \ell \phi+g_{q q^{\prime}}^{\phi} \bar{q}^{\prime} q \phi+\tilde{g}_{f f^{\prime}}^{\phi} \bar{f}^{\prime} f V_{\mathrm{SM}}+\ldots \quad \mathscr{L}_{\psi \phi}=h_{\ell \psi \phi} \bar{\ell} \psi \phi+h_{q \psi \phi} \bar{q} \psi \phi+h_{\ell q \phi} \bar{\ell} q \phi+\ldots
$$

The first terms in $\mathscr{L}_{\phi}$ schematically denote the new cLFV couplings of fermions to an extended gauge and/or scalar sector (with $V_{\mathrm{SM}}$ denoting a SM gauge boson); these can induce loop-level contributions to $\mu-e$ conversion or even tree level exchanges. The t-channel exchanges can be mediated by a new $Z^{\prime}$ boson, with flavour violating couplings to charged leptons, via scalar fermions - as is the case of sneutrinos in R-parity violating supersymmetric (SUSY) models -, or finally, modified couplings of leptons to a SM $Z$ boson, due to the presence of an enlarged Higgs sector (an example being a type III seesaw mechanism). 


\begin{tabular}{lllcc}
\hline Model & $\mu \rightarrow e e e$ & $\mu N \rightarrow e N$ & $\frac{\mathrm{BR}(\mu \rightarrow e e e)}{\mathrm{BR}(\mu \rightarrow e \gamma)}$ & $\frac{\mathrm{CR}(\mu N \rightarrow e N)}{\mathrm{BR}(\mu \rightarrow e \gamma)}$ \\
\hline MSSM & Loop & Loop & $\approx 6 \times 10^{-3}$ & $10^{-3}-10^{-2}$ \\
Type-I seesaw & Loop & Loop & $3 \times 10^{-3}-0.3$ & $0.1-10$ \\
Type-II seesaw & Tree & Loop & $(0.1-3) \times 10^{3}$ & $\mathscr{O}\left(10^{-2}\right)$ \\
Type-III seesaw & Tree & Tree & $\approx 10^{3}$ & $\mathscr{O}\left(10^{3}\right)$ \\
LFV Higgs & Loop & Loop & $\approx 10^{-2}$ & $\mathscr{O}(0.1)$ \\
Composite Higgs & Loop & Loop & $0.05-0.5$ & $2-20$ \\
\hline
\end{tabular}

Table 1: Pattern of the relative predictions for $\mu \rightarrow e$ processes as predicted in several models. Adapted from [25], to which we refer for further details.

The second set of terms in Eq. (3.1), $\mathscr{L}_{\psi \phi}$, corresponds to having cLFV couplings to new fermions and scalars which necessarily carry lepton number. For example, the new states can correspond to sleptons and electroweak gauginos in SUSY models (flavour violating extensions, or SUSY realisations of the seesaw mechanism), heavy right-handed neutrinos and vector bosons in Left-Right models, among many others; the contributions arise in general via higher-order (loop) processes. However, one can also have new tree level (s-channel) contributions, for instance mediated by scalar leptoquarks carrying both lepton and baryon number.

By itself, and other than clearly signaling a NP discovery, the observation of a neutrinoless muon-electron conversion in matter does not offer clear information regarding the nature of the BSM model at work: a vast array of new interactions and fields can be at the source of the transition. In the absence of a direct discovery at colliders (and in all cases, to single out the nature of the new propagators), it is mandatory to study in depth the interplay of different cLFV observables. The synergy of identical transitions between different flavours (e.g. the comparison of $\tau \rightarrow \mu \gamma$ with $\mu \rightarrow e \gamma$ decays, or then $\tau \rightarrow 3 \mu$ with $\mu \rightarrow 3 e$ ) offers insight on the structure of a common cLFV interaction; on the other hand, the comparison of different processes requiring similar flavour violation ( $\mu \rightarrow e \gamma, \mu \rightarrow 3 e$ and $\mu-e$ conversion) could shed light on the nature of the new mediator. To illustrate this, let us carry a comparative discussion of the three seesaw realisations, and then their supersymmetrised versions (SUSY seesaws). The seesaw can be realised via the addition of new fields to the SM content: singlet fermions (type I), scalar triplets (type II) and finally fermion triplets (type III). In the first case, all cLFV transitions (radiative, 3-body decays and muon-electron conversion) are loop processes. For a type II seesaw, 3-body decays can already occur at tree level, while for type III only radiative decays remain a higher order transition. Clearly, each realisation will be associated with peculiar patterns for the observables. For instance, depending on the mass of the heavy neutrinos, in a type I seesaw one can have the ratio of $\mathrm{BRs}, \operatorname{BR}(\mu \rightarrow 3 e) / \mathrm{BR}(\mu \rightarrow e \gamma)$ between $\mathscr{O}\left(3 \times 10^{-3}-0.3\right)$; for a type II seesaw, and again depending on the mass of the mediators, the ratio of rates will differ significantly, in the range $(0.1-3) \times 10^{3}$. Finally, for type III, almost independently of the mass of the fermion triplets, one finds $\operatorname{BR}(\mu \rightarrow 3 e) / \operatorname{BR}(\mu \rightarrow e \gamma) \sim \mathscr{O}\left(10^{3}\right)$. The comparison of the neutrinoless muon-electron conversion rate to that of the radiative muon decay also contributes to further disentangle the scenarios (especially type II vs. type III): one finds $\mathscr{O}(0.1-10), \mathscr{O}\left(10^{-2}\right)$ and $\mathscr{O}\left(10^{3}\right)$, respectively for type I, II and III seesaws. This is summarised on Table 3.1 (adapted from [25]).

For the supersymmetrisation of the type I seesaw, all cLFV processes discussed above remain 
higher order transitions. Radiative decays correspond to slepton-gaugino mediated loops, while 3-body decays and muon-electron conversion can be mediated by penguins or boxes, the first being in general dominant. The flavour conserving interaction with two final state leptons or quarks in the nuclei can be mediated via photon, $Z$ or Higgs exchanges. Should photonic penguins dominate, one expects a correlation between $\operatorname{BR}(\mu \rightarrow e \gamma), \operatorname{BR}(\mu \rightarrow 3 e)$ and $\mathrm{CR}(\mu-e, \mathrm{~N})$ : in general, one has $\mathrm{CR}(\mu-e, \mathrm{~N}) \approx 5 \times 10^{-3} \operatorname{BR}(\mu \rightarrow e \gamma)$, and $\operatorname{BR}(\mu \rightarrow 3 e) \approx 6 \times 10^{-3} \operatorname{BR}(\mu \rightarrow e \gamma)$. In the case in which the Higgs-penguin provides the dominant contributions to muon-electron conversion, the first relation is significantly affected, $\mathrm{CR}(\mu-e, \mathrm{~N}) \approx 0.08-0.15 \mathrm{BR}(\mu \rightarrow e \gamma)$. (See also Table 3.1).

Other NP realisations, such as cLFV Higgs sectors, or composite Higgs scenarios, also lead to peculiar imprints regarding the relations between the different cLFV observables, as can be seen from the two last lines of Table 3.1.

\subsection{Further examples: cLFV, LNV, LFUV and models of NP}

In what follows we provide a few examples of how significant contributions to the different observables discussed in Section 2 arise from BSM constructions. An important point to emphasise is that the NP model responsible for cLFV, etc. need not be the one responsible for neutrino mass generation.

Simplified "toy" models of sterile neutrinos A useful first approach to study muon-related observables in the presence of massive neutrinos is to consider simple, "toy"-model like adhoc constructions in which the SM is minimally extended by at least one massive sterile fermion ${ }^{2}$. For the case of a single additional Majorana sterile state, neutrino mixings are thus encoded into a $4 \times 4$ matrix, parametrised by 6 mixing angles and $6 \mathrm{CP}$ violating phases. The left-handed leptonic mixings correspond to a non-unitary $3 \times 3$ sub-block, and the ensuing modification of charged and neutral lepton currents opens the door to many NP observables in the lepton sector including cLFV, LNV and LFUV. If the (Majorana) sterile state has a mass allowing it to be produced on-shell from meson and tau decays, then one can also have a resonant enhancement of the rates for LNV semileptonic meson and tau decays [26, 27].

Figure 1 illustrates the prospects for both cLFV and LNV of such a minimal SM extension. On the left, we display a comparison of two cLFV muonic observables, neutrinoless muon-electron conversion in nuclei (on the left axis) and the muon decay into 3 electrons (on the right axis), both as a function of the mass of the heavy sterile state, $m_{4}$ [28]. As is manifest, the addition of a single sterile state can be at the source of very large cLFV contributions (especially for sterile masses above the electroweak scale), lying within the sensitivity of future dedicated facilities (Mu2e/COMET and Mu3e). On the right panel we display the rates for the LNV semileptonic kaon decays, $\mathrm{BR}\left(K^{+} \rightarrow \ell_{\alpha}^{+} \ell_{\beta}^{+} \pi^{-}\right)$, again as a function of the mass of the mediating Majorana sterile state. As can be seen, several modes can have large branching fractions, some even already in conflict with experimental bounds [27].

Inverse seesaw: from cLFV to LFUV in the kaon sector The Inverse Seesaw (ISS) is a phenomenologically and theoretically appealing low-scale model for neutrino mass generation. Three

\footnotetext{
${ }^{2}$ These models can be interpreted as a simple phenomenological bottom-up approach, without making any formal assumption on the underlying mechanism of neutrino mass generation, or equivalently that there is no relation between neutrino masses and leptonic mixings.
} 

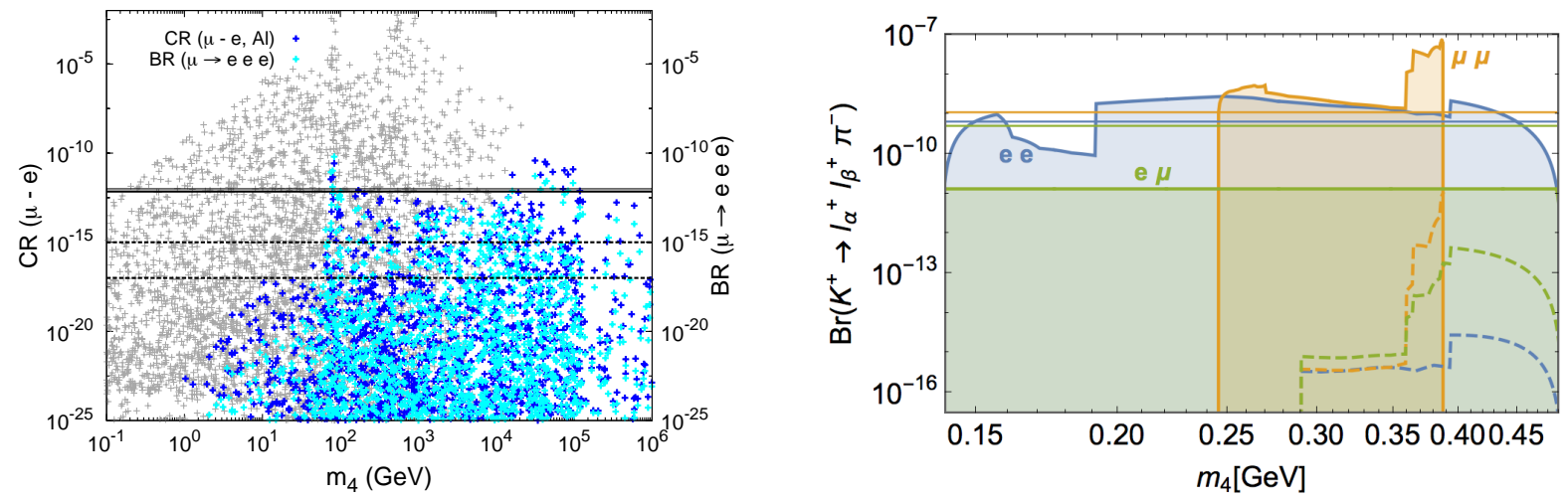

Figure 1: On the left, $\operatorname{CR}(\mu-e)$ and $\operatorname{BR}(\mu \rightarrow 3 e)$ as a function of $m_{4}$, respectively in dark blue (left axis) and cyan (right axis). Thick (thin) solid horizontal lines denotes the current experimental bounds, and dashed lines correspond to future sensitivities to the conversion rate (from [28]). On the right panel, LNV $\mathrm{BR}\left(K^{+} \rightarrow \ell_{\alpha}^{+} \ell_{\beta}^{+} \pi^{-}\right)$, with $\left(\ell_{\alpha}, \ell_{\beta}\right)=(e e, e \mu, \mu \mu)$, as a function of the sterile state mass, $m_{4}$. Coloured curves (surfaces) denote the maximal (allowed) values of the corresponding BRs. From [27].

sets of right-handed neutrinos and extra sterile fermions are added to the SM content in the socalled $(3,3)$ ISS realisation; the new states do not decouple, leading to modified leptonic currents and extensive contributions to many observables. In Fig. 2, we illustrate the prospects for CLFV and LFUV (in leptonic kaon decays). As can be seen from the left panel, in which we display the contributions to the Coloumb-enhanced muonic atom decay and neutrinoless conversion in Nuclei, the contributions in both cases are also within experimental reach [28]. On the right panel we illustrate the ISS contributions to $R_{K, \pi}^{\ell}$. Interestingly, the ISS is one of the few NP models capable of saturating the still sizeable deviation between the SM prediction and observation, $\Delta r_{K, \pi}$, while complying with the stringent bounds from EW data and unitarity violation [29].

Leptoquarks, the $B$-meson decay anomalies and cLFV In recent years, SM extensions via leptoquarks (scalar or vector fields) have been receiving increasing attention, especially in view of their intrinsic potential to address the LFUV $B$-meson decay anomalies. In addition to providing the (required) source of lepton flavour universality violation, these models in general lead to flavour violation. In particular, certain processes - such as the case of meson decays, or neutrinoless muonelectron conversion, can occur at tree-level. The latter muonic observable thus emerges as one of the most severe constraints for this class of BSM constructions. As an illustrative example, we have considered a SM extension via a single vector leptoquark, and 3 generations of vector-like heavy leptons. Similar to the case of additional sterile neutrinos, which leads to non-unitary left-handed leptonic mixings, the mixings of the charged leptons with the heavy vector-like fermions lead to a departure from unitarity, and hence to modified leptoquark-quark-lepton interactions, which in turn successfully allows to address both $R_{K^{(*)}}$ and $R_{D^{(*)}}$ [30]. The model is severely constrained by electroweak precision observables and from cLFV bounds (in particular from $K \rightarrow e \mu$ decays and $\mu-e$ conversion in Nuclei - both occurring at the tree level). Realisations via isosinglet 

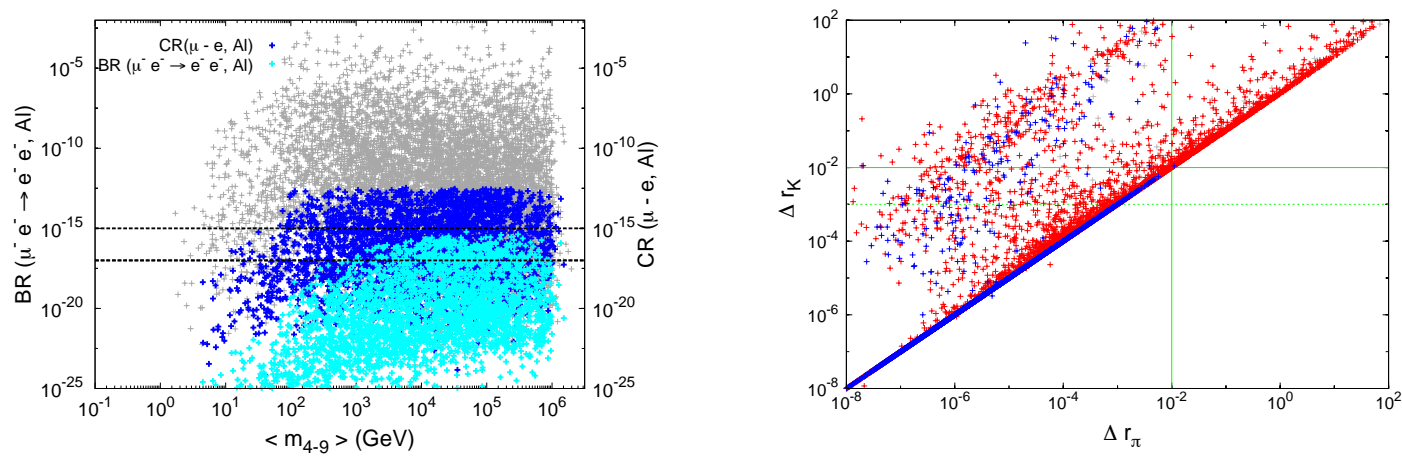

Figure 2: On the left, $\mathrm{BR}(\mu e \rightarrow e e)$ and $\mathrm{CR}(\mu-e)$ as a function of an average sterile mass, respectively in cyan (left axis) and blue (right axis). Dotted lines correspond to future sensitivities (from [28]). On the right ISS contributions to $\Delta r_{K, \pi}$. From [29].

heavy leptons are displayed on the left panel of Fig. 3: constraints from $Z$ decays isolate a small region in which only $R_{D^{(*)}}$ can be accounted for; however these regimes are already excluded by both $K \rightarrow e \mu$ and $\mu-e$ conversion current bounds. Isodoublet heavy leptons do successfully explain both anomalies; interestingly, the regions complying with current cLFV bounds are within sensitivity of future $\mu-e$ conversion experiments, so that the model can be effectively probed by both Mu2e and COMET.
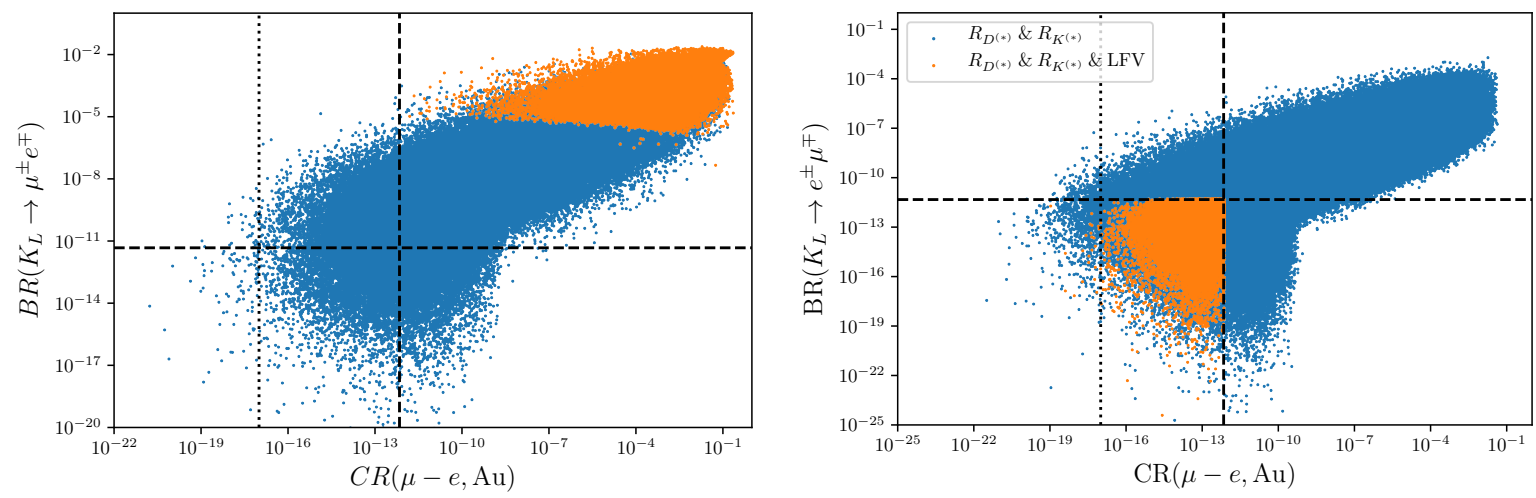

Figure 3: On the left, realisation with isosinglet heavy leptons: blue points comply with $R_{D^{(*)}}$, while orange ones satisfy EW precision data. On the right, extensions via isodoublet heavy leptons: blue points comply with both $R_{K^{(*)}}$ and $R_{D^{(*)}}$, while orange points now denote regions complying with all other bounds. In both cases, dashed lines denote current bounds, and dotted ones a future benchmark sensitivity for $\operatorname{CR}(\mu-e$, $\mathrm{Al}) \sim 10^{-17}$. From [30].

The probing power of muons (be it in the form of cLFV decays, EDMs, $(g-2)_{\mu}$, or other observables) can be studied for numerous models of NP (some discussed in [31]): further examples, beyond those here mentioned, include low scale type I seesaw (and variants), scotogenic models, 
SM extensions via vector-like leptons and extra scalars, SUSY seesaw, extra-dimensional RandalSundrum models, among many others.

\section{Overview and discussion}

Following the discovery of neutrino oscillations, a number of tensions between experimental data and SM expectations has emerged in recent years. Interestingly, all these tensions are nested in lepton related-observables.

Due to their properties, muons are excellent probes of New Physics; moreover, the experimental prospects to have them produced with unprecedented intensity lend hope to confirming the presence of NP in very rare processes, or then via tiny deviations from SM expectations. The future experimental programme offers the possibility to fully explore the probing power of these particles. The synergy of lepton observables can provide information on the the underlying NP model, allowing to falsify it and/or to probe NP scales which are otherwise unreachable. A quanlitative view can be inferred from the so-called SM-effective theory approach: assuming that the new states are sufficiently heavy, their impact can be encoded in higher order, non-renormalisable effective operators, constructed from the SM fields as $\mathscr{L}^{\text {eff }}=\mathscr{L}^{\mathrm{SM}}+\sum_{n \geq 5} \frac{1}{\Lambda^{n-4}} \mathscr{C}^{n} \mathscr{O}^{n}$. For instance, cLFV processes probe combinations $\mathscr{C}^{6} / \Lambda^{2}$. Assuming for simplicity $\mathscr{C}^{6} \sim \mathscr{O}(1)$, an estimation of the NP scales $\Lambda$ probed by a given experiment can be obtained by comparing the expected sensitivity with the observable cast in terms of the effective couplings and new physics scale. As is manifest from Fig. 4 (from [3]), the next generation of lepton (flavour and CPV) experiments is expected to probe significantly higher effective NP scales, as high as $\mathscr{O}\left(10^{5}-10^{7}\right) \mathrm{TeV}$.

The experimental prospects for the coming facilities, especially those dedicated to study lepton observables, is unmatched; hopefully NP will be manifest via one of the many signals (cLFV, LNV, $\mathrm{CPV}, \ldots$ ) being actively searched for.

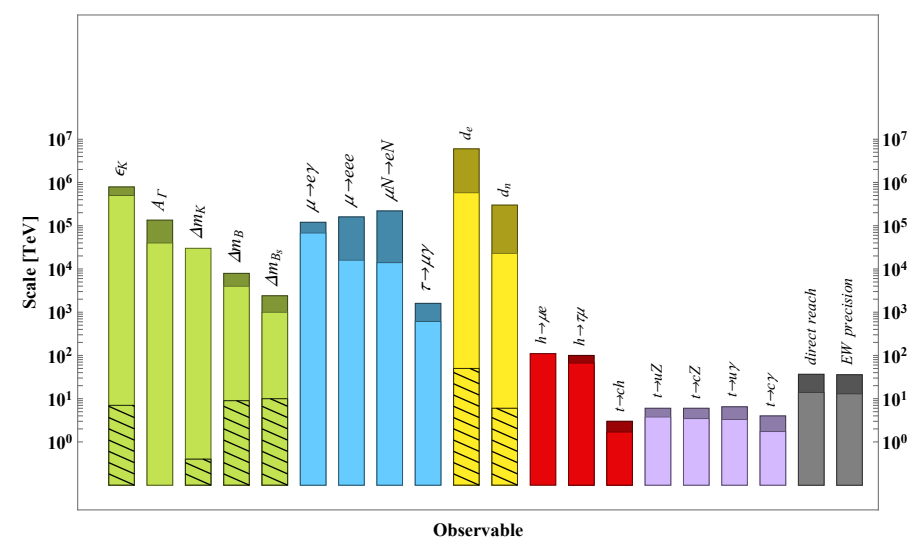

Figure 4: Reach in new physics scale of present and future facilities, from generic dimension six operators, regarding mesons (green), leptons (blue), EDMs (yellow), Higgs flavoured couplings (red) and top quark (purple). The reach of (flavour-blind) direct searches and EW precision measurements is denoted via grey columns. From [3], to which we refer for a full description of the underlying analysis and approximations. 


\section{References}

[1] Y. Kuno, proceedings of "NuFact2019”, PoS 2020.

[2] V. Andreev et al. [ACME Collaboration], Nature 562 (2018) no.7727, 355.

[3] Richard Keith Ellis et al., "Physics Briefing Book : Input for the European Strategy for Particle Physics Update 2020,” arXiv:1910.11775 [hep-ex].

[4] G. W. Bennett et al. [Muon (g-2) Collaboration], Phys. Rev. D 80 (2009) 052008.

[5] G. W. Bennett et al. [Muon g-2 Collaboration], Phys. Rev. D 73 (2006) 072003.

[6] A. Keshavarzi, D. Nomura and T. Teubner, Phys. Rev. D 97 (2018) no.11, 114025.

[7] M. Davier, A. Hoecker, B. Malaescu and Z. Zhang, arXiv:1908.00921 [hep-ph].

[8] D. Nomura, proceedings of "NuFact2019", PoS 2020.

[9] T. Aoyama, T. Kinoshita and M. Nio, Atoms 7 (2019) no.1, 28

[10] V. Cirigliano and I. Rosell, Phys. Rev. Lett. 99 (2007) 231801.

[11] C. Lazzeroni et al. [NA62 Collaboration], Phys. Lett. B 719 (2013) 326.

[12] A. M. Baldini et al. [MEG Collaboration], Eur. Phys. J. C 76 (2016) no.8, 434.

[13] A. M. Baldini et al. [MEG II Collaboration], Eur. Phys. J. C 78 (2018) no.5, 380.

[14] U. Bellgardt et al. [SINDRUM Collaboration], Nucl. Phys. B 299 (1988) 1.

[15] A. Blondel et al., arXiv:1301.6113 [physics.ins-det].

[16] S. Davidson, Y. Kuno and A. Saporta, Eur. Phys. J. C 78 (2018) no.2, 109.

[17] R. Kitano, M. Koike and Y. Okada, Phys. Rev. D 66 (2002) 096002.

[18] W. H. Bertl et al. [SINDRUM II Collaboration], Eur. Phys. J. C 47 (2006) 337.

[19] M. Koike, Y. Kuno, J. Sato and M. Yamanaka, Phys. Rev. Lett. 105 (2010) 121601.

[20] B. Yeo, Y. Kuno, M. Lee and K. Zuber, Phys. Rev. D 96 (2017) no.7, 075027.

[21] B. Pontecorvo, Sov. Phys. JETP 6 (1957) 429.

[22] L. Willmann et al., Phys. Rev. Lett. 82 (1999) 49.

[23] J. Kaulard et al. [SINDRUM II Collaboration], Phys. Lett. B 422 (1998) 334.

[24] R. Abramishvili et al. [COMET Collaboration], arXiv:1812.09018 [physics.ins-det].

[25] L. Calibbi and G. Signorelli, Riv. Nuovo Cim. 41 (2018) no.2, 71.

[26] A. Atre, T. Han, S. Pascoli and B. Zhang, JHEP 0905 (2009) 030.

[27] A. Abada, V. De Romeri, M. Lucente, A. M. Teixeira and T. Toma, JHEP 1802 (2018) 169.

[28] A. Abada, V. De Romeri and A. M. Teixeira, JHEP 1602 (2016) 083.

[29] A. Abada, A. M. Teixeira, A. Vicente and C. Weiland, JHEP 1402 (2014) 091.

[30] C. Hati, J. Kriewald, J. Orloff and A. M. Teixeira, JHEP 1912 (2019) 006.

[31] A. M. Teixeira, presentation at "NuFact2019”, held 26-31 August 2019, Daegu, Korea. 